\title{
Differences in Clinical Manifestations according to the Positivity of Interferon- $\gamma$ Assay in Patients with Intestinal Tuberculosis
}

Hye Jin Jung ${ }^{1,2}$, Young-Ho Kim ${ }^{3}$, You Sun Kim ${ }^{1}$, Seong Yeon Jeong ${ }^{1}$, Sung Won Park ${ }^{1}$, Ji Yeon Seo ${ }^{4}$, Hyemi Jung ${ }^{3}$, Jong Pil $\mathrm{Im}^{4}$, Ji Won Kim${ }^{4}$, Sung Noh Hong ${ }^{3}$, and Kuk Lae Lee ${ }^{4}$

${ }^{1}$ Department of Internal Medicine, Inje University College of Medicine, Seoul, ${ }^{2}$ Department of Health Promotion Center, International St. Mary's Hospital, Incheon, ${ }^{3}$ Department of Internal Medicine, Sungkyunkwan University School of Medicine, and ${ }^{4}$ Department of Internal Medicine, Seoul National University College of Medicine, Seoul, Korea

Intestinal tuberculosis (ITB) remains prevalent in Asia. An interferon- $y$ assay (QuantiFERON-TB gold test [QFT]) is considered to be an effective supplementary tool for diagnosing ITB. We retrospectively analyzed the clinical features of ITB patients based on the initial results of QFT. A total of 109 patients with ITB were enrolled, and 82 patients $(75.2 \%)$ showed positive QFT results. In the QFT-positive group, the mean age ( $44.1 \pm 12.0$ years) was significantly higher than that in the QFT-negative group $(37.0 \pm 14.8, p=0.0096)$. Abdominal pain $(p=0.006)$ and diarrhea $(p=0.030)$ were more frequent in the QFT-negative group. Further, C-reactive protein (CRP) levels were significantly higher in the QFTnegative group $(6.4 \pm 9.9 \mathrm{mg} / \mathrm{dL})$ than in the QFT-positive group $(1.3 \pm 2.3, p<0.001)$. Multivariate analysis confirmed that younger age $(p=0.016)$, diarrhea $(p=0.042)$, and high levels of $\operatorname{CRP}(p=0.029)$ were independent predictors of QFTnegative results in patients with ITB. These results suggest that prior exposure to TB, reflected by QFT positivity, may cause mild inflammation in patients with ITB. (Gut Liver 2016;10:649-652)

Key Words: Intestinal tuberculosis; Interferon-gamma assay; C-reactive protein

\section{INTRODUCTION}

Tuberculosis (TB) remains prevalent worldwide with considerable morbidity and mortality rates. ${ }^{1}$ Currently, one-third of the world's population is infected with TB, demonstrating its resurgence. ${ }^{1}$ Intestinal tuberculosis (ITB) is a form of extrapulmonary $\mathrm{TB}$ that involves the gastrointestinal tracts. ITB is generally rare, accounting for $1 \%$ to 3\% of TB cases, ${ }^{2}$ however, the incidence of ITB in both developing and developed countries has increased steadily in recent 20 years. ${ }^{3}$ It is important to differentiate ITB from inflammatory bowel disease (IBD), especially Crohn's disease (CD) in Asian countries, including China, Taiwan, India, and Korea, because of the increasing prevalence of $\mathrm{CD}$ with a still-high prevalence of ITB. ${ }^{4-7}$ However, it is sometimes challenging to distinguish these two diseases because of their similar clinical manifestations and examination results. ${ }^{7,8}$

Recently, an interferon- $\gamma$ release assay (IGRA) that measures the release of interferon- $\gamma$ (IFN) after stimulation in vitro by Mycobacterium tuberculosis-specific antigens has been used as an aid in diagnosing both latent TB infection (LTBI) and active TB disease. ${ }^{9-11}$ IFN- $\gamma$ plays an important role in control of cellmediated immune reactivity to $M$. tuberculosis. There are two IGRAs, QuantiFERON-TB gold test (QFT) (Cellestis Ltd., Carnegie, Australia) and T-SPOT.TB (TSPOT) (Oxford Immunotec Ltd., Abingdon, UK) tests available and their results are comparable. IGRA also has been utilized in the differential diagnosis of ITB and $\mathrm{CD}^{.}{ }^{12-14}$ In our previous study, the positive rate for the QFT in patients with ITB was significantly higher than it was in patients with CD $(67 \%$ vs $9 \%, p<0.001)$ and we suggested that the QFT is a limited but useful diagnostic aid in the diagnosis of ITB. ${ }^{12}$

However, the clinical implication of the results of QFT in patients with ITB remains unknown. Only two-thirds of ITB patients showed positive QFT results, with the remainder showing negative results. Therefore, we evaluated the clinical differences of ITB patients according to the results of QFT.

Correspondence to: You Sun Kim

Department of Internal Medicine, Seoul Paik Hospital, Inje University College of Medicine, 9 Mareunnae-ro, Jung-gu, Seoul 04551, Korea

Tel: +82-2-2270-0012, Fax: +82-2-2279-4021, E-mail: yousunk69@korea.com

Received on September 4, 2015. Revised on December 12, 2015. Accepted on December 26, 2015. Published online June 13, 2016

pISSN 1976-2283 eISSN 2005-1212 http://dx.doi.org/10.5009/gnl15439

Hye Jin Jung and Young-Ho Kim contributed equally to this work as first authors.

(a) This is an Open Access article distributed under the terms of the Creative Commons Attribution Non-Commercial License (http://creativecommons.org/licenses/by-nc/4.0) which permits unrestricted non-commercial use, distribution, and reproduction in any medium, provided the original work is properly cited. 


\section{MATERIALS AND METHODS}

From April 2007 to July 2013, 109 consecutive patients with ITB were enrolled retrospectively from four hospitals in Korea. They all performed the QFT at the time of ITB diagnosis. We used the Korean diagnostic guideline of ITB, which classifies the ITB into definite or probable ITB. ${ }^{15}$ Definite diagnosis of ITB was made on histopathologic findings or culture results. ${ }^{15}$ In patients suspected of having ITB, those with the characteristic endoscopic findings of ITB or positive QFT results, but without definite evidence of ITB, empirical anti-TB therapy was tried for 2 to 3 months. The probable diagnosis of ITB was made when they showed marked improvement in the clinical and endoscopic findings to empirical anti-TB therapy. ${ }^{15}$ We compared the clinical parameters between the two groups according to the results of QFT including clinical symptoms, extents of colonoscopic involvement, laboratory findings, and existence of extraintestinal TB. This study was approved by the ethics committees of each institution.

The QFT (Cellestis Ltd.) test was performed in two stages according to the manufacturer's instructions. A positive response value of $0.35 \mathrm{IU} / \mathrm{mL}$ was used as the cutoff for the QFT test. If the results showed indeterminate, we regarded the data as negative. Comparisons between the two groups were made using Student t-test, Pearson chi-square test, and Fisher exact test. The multivariate analysis was done using logistic regression analysis. Two tailed p-values below 0.05 were considered statistically significant. We conducted statistical analysis using PASW Statistics 17.0 (SPSS Inc., Chicago, IL, USA).

Table 1. Baseline Characteristics of Patients with Intestinal Tuberculosis

\begin{tabular}{lc}
\hline \multicolumn{1}{c}{ Characteristic } & No. of patients $(\mathrm{n}=109)$ \\
\hline Male sex & $55(50.5)$ \\
Age, yr & $42.6 \pm 14$ \\
Definite diagnosis (n=26) & \\
$\quad$ Caseating granuloma & $6(23.1)$ \\
$\quad$ Tissue AFB positive & $4(15.4)$ \\
$\quad$ Tissue culture positive & $16(61.5)$ \\
Positivity of QFT & $82(75.2)$ \\
Prior active TB history & $15(13.8)$ \\
Extraintestinal TB & $29(26.6)$ \\
Underwent surgery & $6(5.5)$ \\
BCG vaccination & $89(81.7)$ \\
Underlying disease & $11(10.1)$
\end{tabular}

Data are presented as the number $(\%)$ or mean \pm SD. Extraintestinal TB is defined as TB involved outside of the gastrointestinal tract. AFB, acid fast bacilli; QFT, QuantiFERON-TB gold test; TB, tuberculosis; BCG, Calmette-Guérin bacillus vaccine.

\section{RESULTS}

A total of 109 patients (mean age, $42.6 \pm 14$ years; male 55, female 54) were enrolled retrospectively in this study. According to the guideline for ITB diagnosis, ${ }^{15} 26$ (23.9\%) had definite diagnosis of ITB and 83 (76.1\%) had probable diagnosis. Table 1 shows the detailed characteristics of enrolled patients.

When the 109 patients separated into two groups according to the QFT results at the time of ITB diagnosis, 82 patients (75.2\%) showed positive QFT results, whereas 27 (24.8\%) showed negative results. Two patients showed indeterminate results and we grouped these two patients as QFT-negative group. The mean age at the time of diagnosis was $44.1 \pm 12$ years in the QFTpositive group, which is significantly higher than the age of QFT-negative group (37.0 \pm 14.8 years, $\mathrm{p}=0.0096)$. In addition, in the QFT-negative group, patients complained significantly more

Table 2. Clinical Parameters of the Patients with Intestinal Tuberculosis Based on the Initial Results of QFT

\begin{tabular}{|c|c|c|c|}
\hline Parameter & $\begin{array}{l}\text { QFT-positive } \\
\qquad(\mathrm{n}=82)\end{array}$ & $\begin{array}{l}\text { QFT-negative } \\
\qquad(n=27)\end{array}$ & p-value \\
\hline Age, yr & $44.1 \pm 12.0$ & $37.0 \pm 14.8$ & 0.0096 \\
\hline Male sex & $40(48.8)$ & $15(55.6)$ & 0.471 \\
\hline \multicolumn{4}{|l|}{ Clinical manifestation } \\
\hline Abdominal pain & 27 (33.0) & $17(63.0)$ & 0.006 \\
\hline Diarrhea & $14(17.1)$ & $10(37.0)$ & 0.030 \\
\hline Hematochezia & $9(11.0)$ & $5(18.5)$ & 0.329 \\
\hline Fever & $4(4.9)$ & $3(11.1)$ & 1.000 \\
\hline Weight loss & $8(9.8)$ & $4(14.8)$ & 0.102 \\
\hline Nonspecific & $34(41.5)$ & $5(18.5)$ & 0.031 \\
\hline Prior active TB history & $12(14.6)$ & $3(11.1)$ & 0.645 \\
\hline Underlying disease & $9(11.0)$ & $2(7.4)$ & 0.072 \\
\hline Colonoscopic involvement & & & 0.198 \\
\hline Ileocecal area & $28(34.1)$ & 7 (25.9) & \\
\hline Less than 4 segments & $24(29.3)$ & $4(14.8)$ & \\
\hline More than 4 segments & $30(36.6)$ & $16(59.3)$ & \\
\hline \multicolumn{4}{|l|}{ Laboratory finding } \\
\hline Initial hemoglobin, $\mathrm{g} / \mathrm{dL}$ & $13.07 \pm 1.93$ & $13.62 \pm 2.14$ & 0.209 \\
\hline Initial WBC, $/ \mathrm{mm}^{3}$ & $6,387 \pm 1,963$ & $9,287 \pm 4,999$ & $<0.001$ \\
\hline Initial ESR, mm/hr & $30.1 \pm 21.7$ & $31.8 \pm 27.6$ & 0.987 \\
\hline Initial CRP, mg/dL & $1.3 \pm 2.3$ & $6.4 \pm 9.9$ & $<0.001$ \\
\hline Initial albumin, g/dL & $4.16 \pm 0.43$ & $3.96 \pm 0.56$ & 0.181 \\
\hline Granuloma in colonic tissue & 35 (42.7) & 9 (33.3) & 0.095 \\
\hline Extraintestinal TB & $20(24.4)$ & 9 (33.3) & 0.592 \\
\hline
\end{tabular}

Data are expressed as the mean \pm SD or number (\%). Underlying disease means the accompanying liver cirrhosis, diabetes mellitus or malignancy. Extraintestinal TB is defined as TB involved outside of the gastrointestinal tract. Nonspecific symptoms are defined as the symptoms not presumed to be related to intestinal tuberculosis. QFT, QuantiFERON-TB gold test; TB, tuberculosis; WBC, white blood cell; ESR, erythrocyte sedimentation rate; CRP, C-reactive protein. 
Table 3. Logistic Regression Analysis with Intestinal Tuberculosis Based on the Initial Negative Results of QFT

\begin{tabular}{lccc}
\hline & OR & $95 \% \mathrm{CI}$ & $\mathrm{p}$-value \\
\hline Age, yr & 0.947 & $0.90-0.99$ & 0.016 \\
Abdominal pain & 3.896 & $0.78-19.29$ & 0.096 \\
Diarrhea & 3.936 & $1.05-14.70$ & 0.042 \\
Initial WBC, $/ \mathrm{mm}^{3}$ & 1.000 & $1.00-1.00$ & 0.091 \\
Initial CRP, $\mathrm{mg} / \mathrm{dL}$ & 1.146 & $0.914-1.001$ & 0.029 \\
\hline
\end{tabular}

QFT, QuantiFERON-TB gold test; OR, odds ratio; CI, confidence interval; WBC, white blood cell; CRP, C-reactive protein.

Significance at $\mathrm{p}<0.05$ by logistic regression analysis.

about the symptoms of ITB, such as abdominal pain $(\mathrm{p}=0.006)$ and diarrhea ( $\mathrm{p}=0.030$ ), as compared to the QFT-positive group. However, there was no difference in gender $(p=0.471)$, the frequency of accompanying extra-intestinal TB $(p=0.592)$, extents of colonoscopic involvement $(\mathrm{p}=0.198)$, and the presence of granulomas in colonic tissues ( $\mathrm{p}=0.095)$ (Table 2). Eleven patients had the underlying diseases such as liver cirrhosis, diabetes mellitus or malignancy. Nine patients were grouped in the QFT-positive group (11\%) and two patients were QFT-negative group $(7.4 \%)$, without statically significance $(\mathrm{p}=0.072)$ (Table 2$)$. Laboratory tests revealed that C-reactive protein (CRP) levels at the time of ITB diagnosis were significantly lower in the QFTpositive group $(1.3 \pm 2.3 \mathrm{mg} / \mathrm{dL})$ as compared to the QFT-negative group $(6.4 \pm 9.9 \mathrm{mg} / \mathrm{dL}, \mathrm{p}<0.001)$. White blood cell count was also significantly lower in the QFT-positive group $(6,387 \pm 1,963$ $\left./ \mathrm{mm}^{3}\right)$ than QFT-negative group $\left(9,287 \pm 4,999 / \mathrm{mm}^{3}, \mathrm{p}<0.001\right)$. However, no difference was found between groups regarding the levels of erythrocyte sedimentation rate $(p=0.987)$, hemoglobin ( $\mathrm{p}=0.209)$, and albumin ( $\mathrm{p}=0.181$ ) (Table 2). Multivariate analysis revealed that the younger age (OR, 0.947; $\mathrm{p}=0.016$ ), diarrhea (OR, 3.936; $p=0.042$ ), and high level of CRP (OR, 1.146; $\mathrm{p}=0.029$ ) were the independent predictors of QFT-negative results in patients with ITB (Table 3).

\section{DISCUSSION}

Although current ITB patients show mild clinical manifestations, such as lower surgery rate as compared to patients in the 1970 s to 1990 s, $^{16}$ the prevalence of ITB remains high and troublesome in Asian countries. ${ }^{17}$ A recent meta-analysis confirmed that IGRA has good sensitivity (81\%) and specificity (85\%) for the diagnosis of ITB in Asia. The authors suggested that the overall accuracy of IGRA was relatively high for the diagnosis of ITB. ${ }^{13}$ Another meta-analysis also suggested that IGRA has good specificity (87\%) for accurate differential diagnosis of ITB from $\mathrm{CD} .{ }^{18}$ In this study, QFT positivity was seen in 82 patients with ITB (75.2\%), which is similar to our previous study (67\%). ${ }^{12}$ Considering these results, ${ }^{12-14,18}$ we can conclude that IGRA is a useful tool for the diagnosis of ITB.
However, although QFT is a good diagnostic tool for ITB, not all patients with ITB show positive QFT results. In this study, only 75.2\% of ITB patients showed the positive QFT results. To our knowledge, this is the first report evaluating clinical manifestations according to the results of QFT in patients with ITB.

Interestingly, the mean age of QFT-positive group was higher than that of QFT-negative group. In addition, the clinical manifestations were milder in the QFT-positive group considering the low frequency of diarrhea and the findings concerning CRP levels. These results suggested that QFT-positive patients had later-onset development of ITB and had more mild inflammatory disease.

Indeed, most people infected by $M$. tuberculosis do not develop active TB with clinical symptoms. Initial $M$. tuberculosis infection might be eliminated or remain in an inactive and noninfectious state, which is called LTBI. Among them, 2\% to 5\% of patients may develop active TB. IGRA has been used most frequently to detect LTBI. We suppose that ITB also has the same sequence. Although there is no exact data on the incidence and prevalence of ITB in Korea, older individuals are more likely to have LTBI as compared to younger people. That is, among patients with LTBI, the state in which the bacterial replication is maintained at a subclinical level by the immune system, some patients develop to ITB as a result of several factors, such as malnutrition or immune dysfunction. Therefore, we suppose that many cases of ITB develop from LTBI and the possibility that prior exposure to TB may cause mild inflammation in patients with QFT-positive ITB.

QFT has high specificity for the diagnosis of TB because it examines the $M$. tuberculosis specific antigen (ESAT-6 or CFP-10), leading to the lack of cross-reaction with most non-tuberculous mycobacteria (NTM) or Calmette-Gue'rin bacillus vaccine (BCG). Accordingly, a recent meta-analysis reported that IGRA has high specificity for the diagnosis of LTBI particularly in populations that have received BCG vaccinations. ${ }^{19}$ In this study, most ITB patients (81.7\%) had a history of BCG vaccination according to national policy of Korea.

Our study has some limitations. First, we used a small sample size, as we enrolled ITB patients who had undergone QFT at the time of ITB diagnosis. In clinical practice, use of QFT as an aid for the diagnosis of ITB has been popular since 2009 in Korea. Therefore, the small study population is attributed to the lack of usage of QFT in the diagnosis of ITB before 2009. In addition, ITB patients without QFT test were excluded in this study and there could be selection bias. Second, this study was conducted retrospectively, as we were curious as to why some ITB patients showed negative QFT results. A future prospective study will provide more detailed information about the IGRA-negative ITB patients. Also, in vitro experiments on the mechanism underlying the sensitization of T-cells and clinical manifestations of ITB will give us an understanding of the pathophysiology of ITB. Third, we did not consider the factors which can affect on 
the QFT results including the time of blood draw, blood volume, transportation temperature, or incubation time. ${ }^{11}$

In conclusion, QFT is a useful diagnostic tool for the diagnosis of ITB. The QFT-positive group showed mild inflammatory status considering less frequent clinical symptoms and lower levels of CRP as compared to the QFT-negative group. We suggest that prior exposure to TB may cause mild inflammation in patients with ITB.

\section{CONFLICTS OF INTEREST}

No conflict of interest relevant to this article was reported.

\section{ACKNOWLEDGEMENTS}

This study was supported by a grant of the Korean Health Technology R\&D Project, Ministry of Health \& Welfare, Republic of Korea (A120176).

\section{REFERENCES}

1. Dye C. Global epidemiology of tuberculosis. Lancet 2006;367:938940.

2. Horvath KD, Whelan RL. Intestinal tuberculosis: return of an old disease. Am J Gastroenterol 1998;93:692-696.

3. Yamane T, Umeda A, Shimao H. Analysis of recent cases of intestinal tuberculosis in Japan. Intern Med 2014;53:957-962.

4. Logan I, Bowlus CL. The geoepidemiology of autoimmune intestinal diseases. Autoimmun Rev 2010;9:A372-A378.

5. Amarapurkar DN, Patel ND, Rane PS. Diagnosis of Crohn's disease in India where tuberculosis is widely prevalent. World J Gastroenterol 2008;14:741-746.

6. Yang SK, Yun S, Kim JH, et al. Epidemiology of inflammatory bowel disease in the Songpa-Kangdong district, Seoul, Korea, 1986-2005: a KASID study. Inflamm Bowel Dis 2008;14:542-549.

7. Weng MT, Wei SC, Lin CC, et al. Seminar report from the 2014 Taiwan Society of Inflammatory Bowel Disease (TSIBD) Spring Forum (May 24th, 2014): Crohn's disease versus intestinal tuberculosis infection. Intest Res 2015;13:6-10.

8. Makharia GK, Srivastava S, Das P, et al. Clinical, endoscopic, and histological differentiations between Crohn's disease and intestinal tuberculosis. Am J Gastroenterol 2010;105:642-651.

9. Mazurek GH, Jereb J, Lobue P, et al. Guidelines for using the QuantiFERON-TB Gold test for detecting Mycobacterium tuberculosis infection, United States. MMWR Recomm Rep 2005;54:4955.

10. Shim TS. Diagnosis and treatment of latent tuberculosis infection in patients with inflammatory bowel diseases due to initiation of anti-tumor necrosis factor therapy. Intest Res 2014;12:12-19.

11. Pai M, Denkinger CM, Kik SV, et al. Gamma interferon release assays for detection of Mycobacterium tuberculosis infection. Clin Microbiol Rev 2014;27:3-20.

12. Kim BJ, Choi YS, Jang BI, et al. Prospective evaluation of the clinical utility of interferon-gamma assay in the differential diagnosis of intestinal tuberculosis and Crohn's disease. Inflamm Bowel Dis 2011;17:1308-1313.

13. Ng SC, Hirai HW, Tsoi KK, et al. Systematic review with metaanalysis: accuracy of interferon-gamma releasing assay and antiSaccharomyces cerevisiae antibody in differentiating intestinal tuberculosis from Crohn's disease in Asians. J Gastroenterol Hepatol 2014;29:1664-1670.

14. Kim YS, Kim YH, Kim WH, et al. Diagnostic utility of anti-Saccharomyces cerevisiae antibody (ASCA) and interferon-gamma assay in the differential diagnosis of Crohn's disease and intestinal tuberculosis. Clin Chim Acta 2011;412:1527-1532.

15. Kim YS, Kim YH, Lee KM, Kim JS, Park YS; IBD Study Group of the Korean Association of the Study of Intestinal Diseases. Diagnostic guideline of intestinal tuberculosis. Korean J Gastroenterol 2009;53:177-186.

16. Cho JY, Kim YS, Park WW, et al. Clinical features of intestinal tuberculosis in recent ten years (2001-2010). Intest Res 2011 9:12-18.

17. Lee YM, Huh KC, Yoon SM, et al. Incidence and clinical outcomes of clostridium difficile infection after treatment with tuberculosis medication. Gut Liver 2016;10:250-254.

18. Chen W, Fan JH, Luo W, Peng P, Su SB. Effectiveness of interferon-gamma release assays for differentiating intestinal tuberculosis from Crohn's disease: a meta-analysis. World J Gastroenterol 2013;19:8133-8140.

19. Menzies D, Pai M, Comstock G. Meta-analysis: new tests for the diagnosis of latent tuberculosis infection: areas of uncertainty and recommendations for research. Ann Intern Med 2007;146:340354. 